\title{
Berezinskii-Kosterlitz-Thouless phase transitions with long-range couplings
}

\author{
Guido Giachett圈 \\ SISSA and INFN Sezione di Trieste, Via Bonomea 265, I-34136 Trieste, Italy \\ Nicolò Defenu \\ Institute for Theoretical Physics, ETH Zürich, Wolfgang-Pauli-Str. 27, 8093 Zürich, Switzerland \\ Stefano Ruffo \\ SISSA and INFN Sezione di Trieste, Via Bonomea 265, I-34136 Trieste, Italy and \\ Istituto dei Sistemi Complessi, Consiglio Nazionale delle Ricerche, \\ Via Madonna del Piano 10, I-50019 Sesto Fiorentino, Italy \\ Andrea Trombettoni \\ Department of Physics, University of Trieste, Strada Costiera 11, I-34151 Trieste, Italy \\ SISSA and INFN Sezione di Trieste, Via Bonomea 265, I-34136 Trieste, Italy and \\ CNR-IOM DEMOCRITOS Simulation Center, Via Bonomea 265, I-34136 Trieste, Italy
}

\begin{abstract}
The Berezinskii-Kostelitz-Thouless (BKT) transition is the paradigmatic example of a topological phase transition without symmetry-breaking, where a quasi-ordered phase, characterized by a power law scaling of the correlation functions at low temperature, is disrupted by the proliferation of topological excitations above the critical temperature $T_{\mathrm{BKT}}$. In this letter, we consider the effect of long-range decaying couplings $\sim r^{-2-\sigma}$ on this phenomenon. After pointing out the relevance of this non trivial problem, we discuss the phase diagram, which is far richer than the corresponding short-range one. It features - for $7 / 4<\sigma<2$ - a quasi ordered phase in a finite temperature range $T_{c}<T<T_{\mathrm{BKT}}$, which occurs between a symmetry broken phase for $T<T_{c}$ and a disordered phase for $T>T_{\mathrm{BKT}}$. The transition temperature $T_{c}$ displays unique universal features quite different from those of the traditional, short-range XY model. Given the universal nature of our findings, they may be observed in current experimental realizations in $2 D$ atomic, molecular and optical quantum systems.
\end{abstract}

\section{INTRODUCTION}

Two-dimensional interacting systems are well known not to display conventional symmetry breaking transitions at finite temperature, due to the Hohenberg-Mermin-Wagner theorem [1]. Yet, a phase transition may appear driven by topological defects, according to the celebrated Berezinskii-Kosterlitz-Thouless (BKT) mechanism [2]. In the presence of long-range interactions the Hohenberg-Mermin-Wagner theorem no longer holds and local order parameters, such as the magnetization [3], may have a non-zero expectation value. The general question addressed by this Letter is the fate of the BKT transition when the range of the interactions is increased. The Sak's criterion 4 provides an argument for understanding whether the long-range, power law coupling $\sim 1 / r^{d+\sigma}$ in the classical $O(N)$ model affects criticality. It can be formulated as follows: at low momenta the short-range (SR) and long-range (LR) critical two-points functions behave as

$$
p^{-2+\eta_{\mathrm{sr}}} \quad \text { vs } \quad p^{-\sigma}
$$

respectively, where $\eta_{\mathrm{sr}}$ is the anomalous dimension of the SR limit. Therefore, one can define a critical value of the range of the interactions, $\sigma_{*}=2-\eta_{\mathrm{sr}}$, such that, for $\sigma>\sigma^{*}$, the critical behavior is not affected by LR. The validity of Sak's criterion for the classical $O(N)$ models has been the subject of considerable scrutiny. Indeed, numerical evidences supporting (or rejecting) the Sak's result are notoriously hard to obtain [5 7. Intense theoretical investigations both via MC simulations [5, 8, 9], renormalization group (RG) theory [10 12] and conformal bootstrap 13 appeared all to confirm the validity of Sak's conjecture for the LR-SR crossover so that it is fair to conclude that the criterion has been a useful tool to understand the critical behaviour of LR interacting systems [14 17. The criterion, is believed to apply to all symmetry breaking transition in $d \geq 2$. The status of the $d=2$ classical XY model is rather different, and only few results (later commented) are known. The main reasons are

i) The Sak criterion cannot be straightforwardly applied, since in the SR limit the critical behavior is not described by a single RG fixed point, but rather by a whole line of fixed points with a temperature-dependent exponent $\eta_{\mathrm{sr}}$.

ii) Numerically, the large number of non-vanishing couplings, coming form the LR nature of the interaction, along with the logarithmic scaling typical of $2 D$ systems (the so-called "Texas state argument" [18]) make the study of the $2 D \mathrm{XY}$ universality notoriously challenging.

iii) In the nearest-neighbours $2 D \mathrm{XY}$ model, the classical treatment takes advantage of the duality construction [19, through which one can famously relate the model to the Coulomb gas 20, 21, or the sine-Gordon model [21, 22. However, this is no longer the case already for next-to-nearest-neighbors couplings.

iv) It is known that in the SR limit, the physics of the $2 D$ classical XY model can be related to the one of the $1 D$ 
quantum XXZ model via its transfer matrix [23].

This approach is based on the mapping to hard-core bosons, and therefore to the XXZ model, and cannot be straightforwardly applied to the the case of $X Y$ LR interactions, as one should show the RG irrelevance of terms violating the hard-core condition. Moreover, let us remark that $2 D$ boson gas at finite temperature with (isotropic) $1 / r^{3}$ densitydensity interaction do exhibit a BKT transition [24; but this interaction corresponds to a quantum 1D XXZ in which only in the $z-z$ interaction is long range.

v) Finally, we observe that the treatment of the SR XY model in $2 D$ is very much simplified by the introduction of the Villain model, 25, 26, which can be mapped exactly onto the Coulomb gas, and shares the same universality class of the SR $X Y$ model. The physical reason of their connection in the SR regime, is that the (gapped) amplitude fluctuations of the corresponding $O(2)$ action are irrelevant [27. Thus, once the periodic nature of the phase is taken care of, all the relevant information is present in the theory. However, in the LR regime the interplay between amplitude and phase fluctuations cannot be neglected and it is not known whether they still share the same universality class.

Despite these difficulties, the study of LR XY model is of great interest: first, since its introduction, the BKT mechanism [28 31] has been found to quantitatively describe the universal scaling appearing in several $2 D$ systems with $U(1)$ symmetry, ranging from thin ${ }^{4} \mathrm{He}$ films [32] to quasi-2D layered superconductors [33 37, exciton-polariton systems [38, cold atoms in $2 D$ traps [39, 40] and $2 D$ electron gases at the interface between insulating oxides in artificial heterostructures 41,43]. Apart from these experimental realizations, topological defects are expected to be relevant in several natural phenomena outside the condensed matter realm, such as DNA tangling or stripe formation 44 46. To understand how $\sigma_{*}$ is modified, is then a crucial question in all the cases in which a LR tail of the interaction can be added or tuned, especially because the spin-wave interaction term, already present in the SR case, may destroy, partially or totally, the topological nature of the phase transition. Moreover, the physics of LR interacting systems has recently experienced a new wave of interest, due to the current experimental realizations on atomic, molecular and optical (AMO) systems. In particular, trapped ions [47, 48, Rydberg gases [49] and optical cavities [50, 51] allowed the observation of plenty of exotic equilibrium and dynamical phenomena induced by LR interactions, including entanglement and correlations propagation [52, 53, dynamical phase transitions [54, 55, time crystals [54, 56, 57, and defect scaling [58, 59. These experimental results stimulated an impressive theoretical activity to characterize the equilibrium and dynamical critical scaling induced by LR interactions in a wide variety of different systems [17, 60,65]. Despite this outpouring theoretical activity and the long-standing relation between topological scaling and LR interactions, the possible corrections induced by power-law decaying couplings to the topological BKT scaling remain an open question, testable in experiments.

\section{MODEL \& PRELIMINARIES}

We consider a system of planar rotators on a $2 D$ lattice of spacing $a$, described by the Hamiltonian:

$$
\beta H=\frac{1}{2} \sum_{\mathbf{i}, \mathbf{j}} J_{|\mathbf{i}-\mathbf{j}|}\left[1-\cos \left(\theta_{\mathbf{j}}-\theta_{\mathbf{i}}\right)\right]
$$

where $\mathbf{i}, \mathbf{j} \in \mathbb{Z}^{2}$ and $J_{|\mathbf{i}-\mathbf{j}|}$ has a power-law tail: $J_{|\mathbf{i}-\mathbf{j}|} \sim \frac{g}{|\mathbf{i}-\mathbf{j}|^{2+\sigma}}$ for $|\mathbf{i}-\mathbf{j}| \gg 1$. The exponent $\sigma$ is assumed positive in order to ensure additivity of the thermodynamic quantities [66. For the following arguments the specific form of the couplings is not important, as long as that there are no frustration effects nor competing interactions.

Let us now summarize what we do know for sure about the LR XY model (2):

a) For $\sigma<2$, at low enough temperatures, the system magnetizes, as rigorously proven in 3 . MC simulations at $\sigma=1$ indicate an order-disorder transition and no BKT phase at finite temperature [67. Moreover,one could expect that For $\sigma \leq 1$ the critical exponents of the ferro-paramagnetic transition are expected to be mean-field [11.

b) In agreement with a), the spin-wave theory in which the cosine is expanded to the quadratic order, without imposing the periodicity, as in the original Berezinskii calculation [28, does also magnetize for $\sigma<2$, since the contribution of the spin fluctuations is of the form $\int d^{2} q / q^{\sigma}$ and thus infrared finite.

c) An upper bound for $\sigma_{*}$ has to be $\sigma_{*}=2$, i.e. for sure there is BKT for $\sigma>2$, as one can deduce even from the Sak's argument since $\eta$ is positive. This result is supported by the self-consistent harmonic calculation recently presented in [68, which anyway is unable to make even qualitative predictions for $\sigma<2$.

\section{EFFECTIVE MODEL}

We decompose the coupling as $J_{|\mathbf{i}-\mathbf{j}|}=J_{|\mathbf{i}-\mathbf{j}|}^{S}+g|\mathbf{i}-\mathbf{j}|^{-(2+\sigma)}$ where $J^{S}$ is a SR term taking into account the smalldistances behavior of the coupling. At low temperatures, the spin direction varies smoothly from site to site and, as a consequence, we can expand the SR term for small phase differences as $\cos (\theta(\mathbf{x}+\mathbf{r})-\theta(\mathbf{x})) \sim 1-|\nabla \theta|^{2} / 2$. The same, 
however, it is not automatically true for the LR term, since far-away pairs, whose phase difference is not necessarily small, give a significant contribution to the Hamiltonian.

These considerations allow us to write a continuous version of the Hamiltonian in Eq. (2) in terms of the field $\theta(\mathbf{x})$, namely the Euclidean action

$$
S[\theta]=\frac{J}{2} \int d^{2} x|\nabla \theta|^{2}+S_{\mathrm{LR}}
$$

where the LR part can be written as

$$
S_{\mathrm{L} R}=-\frac{g}{2 \gamma_{2, \sigma}} \int d^{2} x\left(\cos \theta \nabla^{\sigma} \cos \theta+\sin \theta \nabla^{\sigma} \sin \theta\right),
$$

with $\gamma_{2, \sigma}=2^{\sigma} \Gamma\left(\frac{1+\sigma}{2}\right) \pi^{-1}\left|\Gamma\left(-\frac{\sigma}{2}\right)\right|^{-1}$, by using the definition of (bulk) fractional derivative given in Appendix A. The first and the second term in Eq. (3) account for the short- and long-range contributions respectively, with $J \sim 1 / T$ and $g \sim 1 / T$ being the temperature dependent couplings. Notice that the result would be different for a quantum $1 D$ chain with LR interactions, where interactions are still SR along the imaginary time axis [69].

If $g=0$, by following the usual duality procedure [26, one can take into account the periodic nature of the field $\theta$ in Eq. (3) by isolating the topological configurations and introducing the vortex fugacity $y=\exp \left(-\varepsilon_{c}\right)$, being $\varepsilon_{c}$ the corresponding core energy. This, in turn, leads to the Kosteritz-Thouless RG equations [26, 29, 30, 70, (see [71, 72. for textbook presentations) which feature a line of stable Gaussian fixed points for $y=0$ and $J>\frac{2}{\pi}$, describing the power-law scaling observed in the low-temperature BKT phase. For $g$ small enough, we expect to have then a continuum theory described by the three parameters $J, g$ and $y$.

In order to explore the effects of LR interactions, we deform the traditional BKT fixed-points theory with the non-local operator in the second term of Eq. (3). Since only those fixed-points which are stable under topological perturbation correspond to an infra-red (IR) limit of the SR BKT theory, we can restrict ourselves to the region in which the topological excitations are irrelevant $\left(J>\frac{2}{\pi}\right)$. The relevance of the LR perturbation depends on the scaling dimension $\Delta_{g}$ of the coupling $g$, which is defined according to the asymptotic behavior $g_{\ell} \approx \exp \left(\Delta_{g} \ell\right)$ for $\ell \gg 1$, where as usual in the BKT literature, we put $\ell=\ln (r / a)$. On the other hand, due to the Gaussian nature of the measure,

$$
\left\langle\cos \left(\theta(\mathbf{x})-\theta\left(\mathbf{x}^{\prime}\right)\right)\right\rangle=e^{-\frac{1}{2}\left\langle\left(\theta(\mathbf{x})-\theta\left(\mathbf{x}^{\prime}\right)\right)^{2}\right\rangle}=\left|\mathbf{x}-\mathbf{x}^{\prime}\right|^{-\eta_{\mathrm{sr}}(J)}
$$

where $\eta_{\mathrm{sr}}(J)=\frac{1}{2 \pi J}$ is the exponent of the SR two-point function, 26, 29, 30, Following Eq. (5), the scaling dimension of the LR term reads

$$
\Delta_{g}=2-\sigma-\eta_{\mathrm{sr}}(J)
$$

so that the LR perturbation becomes relevant only if $\sigma<2-\eta_{\mathrm{sr}}(J)$, similarly to the traditional spontaneous symmetry breaking (SSB) case [11, but with a temperature-dependent anomalous dimension. This confirms that for $\sigma>2$ the LR perturbation is always irrelevant, as expected.

Let us now consider the case $\sigma<2$. There, the LR perturbation becomes relevant at small temperatures, since $\eta_{\mathrm{sr}} \simeq 0$ for $T \simeq 0$. Since $\eta_{s r}$ in Eq. $(6)$ is the one of the SR unperturbed theory, we can apply the results of the traditional BKT theory [3] as long as the LR perturbation is not relevant. In particular we know that topologicaly excitations are irrelevant for $\eta_{s r}<1 / 4$, so that in the range $7 / 4<\sigma<2$, a subset of the BKT fixed points remains stable and we have quasi-long-range order (qlro) for a certain temperature window. This result is rather non trivial, since in SSB transitions the traditional Sak's result 4 predicts the irrelevance of LR couplings at all temperatures for $\sigma>2-\eta_{\mathrm{sr}}$.

\section{RG FLOW}

These results may be confirmed by deriving the flow equations for the LR term at the leading order in $g$ for $y=0$, obtaining (see Appendix B):

$$
\begin{aligned}
\frac{d g_{\ell}}{d \ell} & =\left(2-\sigma-\eta_{\mathrm{sr}}\left(J_{\ell}\right)\right) g_{\ell} \\
\frac{d J_{\ell}}{d \ell} & =c_{\sigma} \eta_{s r}\left(J_{\ell}\right) g_{\ell}
\end{aligned}
$$

where $c_{\sigma}=\frac{\pi}{2} a^{2-\sigma} \int_{1}^{\infty} d u u^{1-\sigma} \mathcal{J}_{0}(2 \pi u)$ and $\mathcal{J}_{0}(x)$ is the zeroth order Bessel function of the first kind. As shown in Appendix B the above result is reliable as long as $a^{2-\sigma} g_{\ell} \ll J_{\ell}$ or, equivalently, as long as $\frac{d J}{d \ell} \ll J_{\ell}$. As expected, we see that the flow equations 29) support a line of SR fixed points $g=0$ which becomes unstable for $\eta_{\text {sr }}(J)<2-\sigma$. As long as our hypothesis of small $g$ holds, we can explicitly identify the form of the flow trajectories of Eqs. 29):

$$
g_{\ell}(J)=\frac{\pi(2-\sigma)}{c_{\sigma}}\left[\left(J_{\ell}-J_{\sigma}\right)^{2}+k\right]
$$




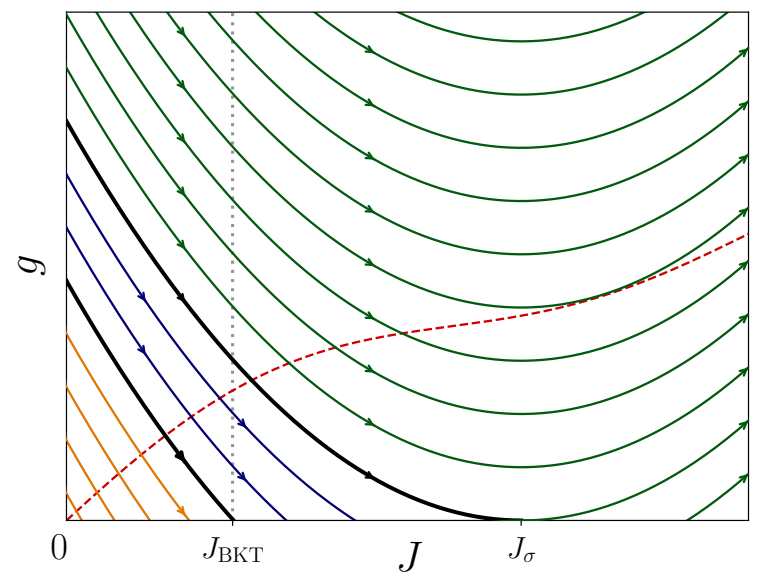

Figure 1: Sketch of the RG flow lines for $\frac{7}{4}<\sigma<2$ in the $y=0$ plane. The dashed red line is a possible realization of the physical parameters line, from which the flow starts, as the temperature is varied. On the right/left of the gray dotted line the vortex fugacity $y$ is irrelevant/relevant $\left(\dot{y}_{\ell} / y_{\ell} \gtrless 0\right)$. The two separatrices (bold black lines) divide the flow in three regions: a high-temperature region (orange, the flow ends up in the disordered phase), an intermediate one (blue, the flow reaches a $g=0$ fixed point) and the low-temperature region (green, the LR perturbation brings the system away from the critical line).

where $k$ is a real number and $J_{\sigma}=\frac{1}{2 \pi(2-\sigma)}$. The sign of $k$ divides the trajectories which met the fixed point $g=0$ and those which do not, the first ones ending at (starting from) the fixed point line for $J \leq J_{\sigma}\left(J>J_{\sigma}\right)$. The separatrix corresponds to the semi-parabola with $k=0, J \leq J_{\sigma}$. For $k>0 \mathrm{~g} \rightarrow \infty$, showing the existence of a new low-temperature phase, where LR interactions are relevant. The critical temperature $T_{c}$, below which this new phase appears, is such that $\eta_{\mathrm{sr}}\left(J_{c}\right)>2-\sigma$.

Since, as in the traditional BKT calculation [29], Eqs. 29] were derived for small $g$ and $y$, its use for $T<T_{c}$ is in principle not justified, since LR interactions are relevant and $g_{\ell}$ grows indefinitely. However, let us notice that the scaling of $g_{\ell}$ with $T$ for $T \rightarrow T_{c}^{-}$can be reliably predicted from Eqs. 29 , since in this limit the flow spends a divergent amount of RG time $\ell$ in the vicinity of the line of fixed points $g=0$. This scaling is derived in Appendix C. Moreover, we can guess the infrared form of the action in the low temperature phase by observing that the rigorous result of Ref. [3] implies that for $T<T_{c}$ the system displays finite magnetization and, then, phase fluctuations are limited even at large distances. Therefore, the expansion of the trigonometric function in Eq. (3) is justified leading to an action of the form

$$
S_{g}=-\frac{\bar{g}}{2} \int d^{2} x \theta \nabla^{\sigma} \theta
$$

where $\bar{g}=g \gamma_{2, \sigma}^{-1}$. Being the above action quadratic, the properties of this exotic low temperature phase can be worked out: in particular the scaling of the magnetization for $T \rightarrow T_{c}^{-}$is found to be (see Appendix C for details)

$$
\ln m \sim-e^{B\left(T_{c}-T\right)^{-1 / 2}}
$$

where $B$ is a non universal constant. Since all the derivatives of $m$ with respect to $T$ vanish at $T=T_{c}$ (essential singularity), and since $m$ is linked to the derivative of the free energy with respect to the external field, we have that the phase transition between the ordered and disordered phase is actually of infinite order. Moreover, the connected correlation functions have a power-law decay for $T<T_{c}$ given by $\langle\mathbf{S}(\mathbf{r}) \cdot \mathbf{S}(\mathbf{0})\rangle_{c} \sim \frac{1}{r^{2-\sigma}}$, where $\mathbf{S}(\mathbf{r})=\left(\cos \theta_{\mathbf{r}}, \sin \theta_{\mathbf{r}}\right)$.

We have so far assumed $y=0$; let us now consider the effect of topological excitations. At leading order in both $g$ and $y$ the two perturbations remain independent and, since the vortices contribute to the $J_{\ell}$ flow only beyond leading order in $y$, Eqs. 29) are unchanged. Moreover, one has $\frac{d y_{\ell}}{d \ell}=\left(2-\pi J_{\ell}\right) y_{\ell}$ valid up to second order terms in $y_{\ell}$ and $g_{\ell}$. Then, in agreement with what we stated above, as long as $\frac{7}{4}<\sigma<2$, the temperature range $T$ between $T_{c}$ and $T_{\mathrm{BKT}}$ of the line of fixed points $g=y=0$ remains stable under both topological and LR perturbations. In the low-temperature phase instead, it is natural to suppose $y$ to be irrelevant, due to the fact that a non-negligible LR coupling increases the cost of, highly non-local topological excitations. This idea is made rigorous in Appendix D where the interaction energy between vortices in the low temperature phase is computed, and it is shown that they cannot proliferate.

Summarizing, for $\sigma \in(7 / 4,2)$ we find three phases: $i)$ an ordered phase for $T<T_{c}$ with finite magnetization and temperature independent power-law correlation functions ii) an intermediate BKT phase for $T_{c}<T<T_{\mathrm{BKT}}$, where 


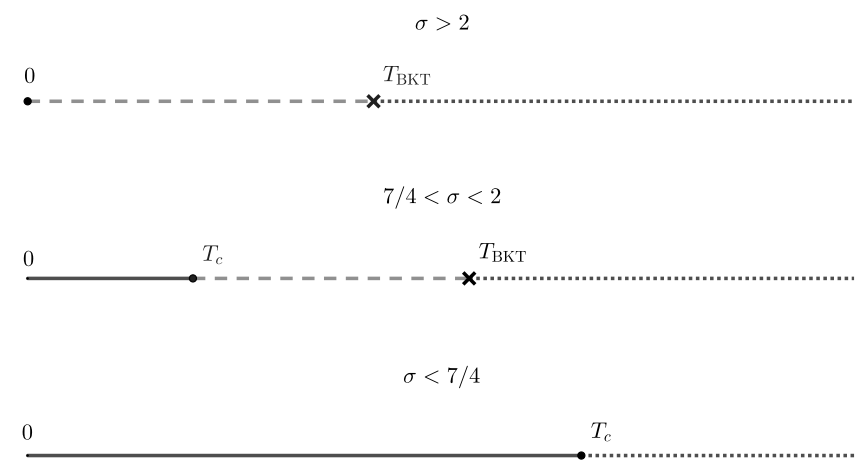

Figure 2: Sketch of the possible phases of the model: ordered with magnetization (solid black), BKT qlro (dashed light gray), disordered (dashed dark gray). If $\sigma>2$ we find the usual SR phenomenology with a BKT phase transition. For $\sigma<2$ an ordered phase appears at low-temperatures, the BKT qlro phase disappearing for $\sigma<\frac{7}{4}$.

the magnetization vanishes and the exponent of the two-point correlation function depends on $T$ iii) a disordered phase for $T>T_{\mathrm{BKT}}$. Due to the LR character of the interactions, also the high-temperature phase displays powerlaw decaying two-point functions $\langle\mathbf{S}(\mathbf{r}) \cdot \mathbf{S}(0)\rangle \sim r^{-2-\sigma}, 74-76$. As $\sigma \rightarrow 7 / 4^{+}$the critical temperature $T_{c}$ reaches $T_{B K T}$ from below. Therefore, for $\sigma<7 / 4$, the whole BKT line fixed points becomes unstable either with respect to topological or LR perturbations and the intermediate phase vanishes, leaving only a SSB phase transition. However, our approach cannot reliably be used to fully characterize this transition: as $T$ approaches $T_{c}$ from below, the RG flow slows down close to the $g=0, J=J_{\sigma}$ fixed point. Since for $\sigma<\frac{7}{4} J_{\sigma}<J_{\mathrm{BKT}}, y$ grows indefinitely, away from the $y \ll 1$ regime. Our results are summarized in Fig. 2 .

\section{CONCLUSIONS}

We have shown that the introduction of long-range (LR) power decaying couplings in the $2 D$ XY model Hamiltonian produces a rich phase diagram, different from the short-range (SR) case [29] and from the one of $O(N)$ LR systems [4]. Remarkably, for $7 / 4<\sigma<2$, the system displays both BKT qlro in the temperature interval $T_{c}<T<T_{\mathrm{BKT}}$ and actual long-range order for $T<T_{c}$.

The introduction of complex interaction patterns in systems with $U(1)$ symmetry is known to generate exotic critical features, as in the anisotropic 3D XY model [77, coupled XY planes [78, 2D systems with anisotropic dipolar interactions [79, 80] or four-body interactions [81, and high-dimensional systems with Lifshitz criticality [82, 83] The present work constitutes a further milestone along this path, as it identifies a peculiar critical behavior, namely a non-analytic exponential vanishing of the order parameter, that eludes the current classification of universal scaling behaviors 84 .

Our predictions may be tested in several low dimensional AMO systems. It would be interesting to perform extensive numerical simulations in order to observe the scaling of the critical quantities, and especially the magnetization, close to the low-temperature endpoint of the BKT line in the regime $7 / 4<\sigma<2$. These simulations will be useful to classify this unprecedented transition and to investigate possible corrections near the $\sigma=7 / 4$ endpoint due to higher-order effects caused by spin-wave excitations 85 . Further investigation is also needed to compare our results with the LR diluted model studied in [86, 87]. In this model, at $\sigma=1.875$, the numerical simulations presented in [87] do not find any intermediate BKT region, but the general question whether the $2 D$ LR diluted XY model and the $2 D$ LR non-diluted one have the same phase diagram remains open.

Our results have also implications for LR quantum XXZ chains [69, 88, 89]. One would need to perform the exact mapping of the classical $2 D$ LR XY model to an effective $1 D$ quantum model, following the calculation presented in 23 and valid for the classical $2 D \mathrm{SR}$ XY model. If the non-local/LR terms violating the hard-core boson condition can be shown to be irrelevant, then one could put in correspondence our phase diagram with that of the LR quantum XXZ chains having LR couplings both for $x-y$ and $z-z$ terms 69 . This seems to be confirmed by the similar structure of the RG flow equations of 69] with our Eqs. 29 taken at low temperatures. If this is the case, then the two lines, black and white, of Fig.1 of 69 would merge in a point, with the XY phase disappearing, corresponding to our $\sigma=7 / 4$ point. Finally, we mention that it would be interesting to study in detail the phase diagram of the $2 D$ LR Villain model for $\sigma<2$. 


\section{ACKNOWLEDGMENT}

Valuable discussions with G. Parisi, F. Ricci-Tersenghi and A. Scardicchio are gratefully acknowledged. N.D. and A.T. also acknowledges useful discussion with M. Ibáñez Berganza. This work is supported by the Deutsche Forschungsgemeinschaft (DFG, German Research Foundation) under Germany's Excellence Strategy EXC2181/1-390900948 (the Heidelberg STRUCTURES Excellence Cluster). This work is supported by the CNR/HAS (Italy-Hungary) project "Strongly interacting systems in confined geometries". This work is part of MUR-PRIN2017 project "Coarse-grained description for non-equilibrium systems and transport phenomena (CONEST)" No. 201798CZL whose partial financial support is acknowledged.

\section{APPENDIX}

\section{A. Defintion of the fractional Laplacian}

Given a real parameter $\sigma \in(0,2)$, one can define the fractional Laplacian of order $\sigma$ of a function $f(\mathbf{x}): \mathbb{R}^{d} \rightarrow \mathbb{R}$ as:

$$
\nabla^{\sigma} f(\mathbf{x}) \equiv \gamma_{d, \sigma} \int d^{d} r \frac{f(\mathbf{x}+\mathbf{r})-f(\mathbf{x})}{r^{d+\sigma}},
$$

where $\gamma_{d, \sigma}=\frac{2^{\sigma} \Gamma\left(\frac{d+\sigma}{2}\right)}{\pi^{d / 2}\left|\Gamma\left(-\frac{\sigma}{2}\right)\right|}$ and $r=|\mathbf{r}|$. Another expression for this quantity can be derived in terms of the Fourier transform, $f(\mathbf{q})$, of $f(\mathbf{x})$ :

$$
\nabla^{\sigma} f(\mathbf{x})=-\gamma_{d, \sigma} \int d^{d} q f(\mathbf{q}) e^{i \mathbf{q} \cdot \mathbf{x}} \int d^{d} r \frac{1-e^{i \mathbf{q} \cdot \mathbf{r}}}{r^{d+\sigma}}
$$

Since

$$
\int d^{d} r \frac{1-e^{i \mathbf{q} \cdot \mathbf{r}}}{r^{d+\sigma}}=\gamma_{d, \sigma}^{-1} q^{\sigma}
$$

we find the alternative definition:

$$
\nabla^{\sigma} f(\mathbf{x})=-\int d^{d} q q^{\sigma} f(\mathbf{q}) e^{i \mathbf{q} \cdot \mathbf{x}}
$$

In our case $d=2$ and we have to evaluate the quantity:

$$
\int d^{2} x \int_{r>a} \frac{d^{2} r}{r^{2+\sigma}}[1-\cos (\theta(\mathbf{x})-\theta(\mathbf{x}+\mathbf{r}))]
$$

For $\sigma<2$, one can actually disregard the contribution coming from the lattice spacing $a$, since it would just result in a correction of the short-range term. Then, through trivial trigonometric manipulations we can write the above expression as:

$$
\begin{aligned}
& \int d^{2} x \cos \theta(\mathbf{x}) \int \frac{d^{2} r}{r^{2+\sigma}}[\cos \theta(\mathbf{x})-\cos \theta(\mathbf{x}+\mathbf{r})] \\
+ & \int d^{2} x \sin \theta(\mathbf{x}) \int \frac{d^{2} r}{r^{2+\sigma}}[\sin \theta(\mathbf{x})-\sin \theta(\mathbf{x}+\mathbf{r})] .
\end{aligned}
$$

Finally, using the definition 11 of the fractional derivative, we get

$$
-\gamma_{2, \sigma}^{-1} \int d^{2} x\left(\cos \theta \nabla^{\sigma} \cos \theta+\sin \theta \nabla^{\sigma} \sin \theta\right)
$$

which justifies the alternative form of the long-range term given in the main text as Eq.(4).

\section{B. Renormalization group for $y=0$}

We will now derive the set of RG equations (7) given in the main text, valid for $y=0$. We then start form the action written in the form

$$
S[\theta]=\int d^{2} x\left(\frac{J_{\ell}}{2}|\nabla \theta|^{2}+\frac{g_{\ell}}{2} \int_{r>a} \frac{d^{2} r}{r^{2+\sigma}}\left[1-\cos \left(\Delta_{\mathbf{r}} \theta(\mathbf{x})\right)\right]\right)
$$


where, as in the main text, $\Delta_{\mathbf{r}} \theta(\mathbf{x})=\theta(\mathbf{x}+\mathbf{r})-\theta(\mathbf{x})$, and compute the flux perturbatively around $g=0$. The field is split into fast and slow modes with respect to the momentum cutoff $\Lambda=\frac{2 \pi}{a}$, namely $\theta=\theta^{>}+\theta^{<}$with

$$
\begin{aligned}
& \theta^{<}(\mathbf{x})=\int_{q<\Lambda e^{-d \ell}} \frac{d^{2} q}{(2 \pi)^{2}} \theta(\mathbf{q}) e^{i \mathbf{q} \cdot \mathbf{x}} \\
& \theta^{>}(\mathbf{x})=\int_{\Lambda>q>\Lambda e^{-d \ell}} \frac{d^{2} q}{(2 \pi)^{2}} \theta(\mathbf{q}) e^{i \mathbf{q} \cdot \mathbf{x}}
\end{aligned}
$$

where $\ell=\ln (r / a)$. If we assume the interacting long-range term in Eq. 18 to be small with respect to the quadratic one, we can perform the integration perturbatively. It is easy to see that this is possible if $g a^{2-\sigma}<<J$. Under this assumption then we integrate out the fast modes, expanding the partition function in cumulants of the non-Gaussian part $S_{g}$ :

$$
S_{\mathrm{eff}}\left[\theta^{<}\right]=S_{0}\left[\theta^{<}\right]+\left\langle S_{g}\right\rangle_{>}+O\left(g^{2}\right)
$$

Writing $\cos \left(\Delta_{\mathbf{r}} \theta\right)=\cos \left(\Delta_{\mathbf{r}} \theta^{>}\right) \cos \left(\Delta_{\mathbf{r}} \theta^{<}\right)+\sin \left(\Delta_{\mathbf{r}} \theta^{>}\right) \sin \left(\Delta_{\mathbf{r}} \theta^{<}\right)$, one sees that only the first term will give a contribution. Then, up to additive constants we have:

$$
\left\langle S_{g}\right\rangle_{>}=\frac{g_{\ell}}{2} \int d^{2} x \int \frac{d^{2} r}{r^{2+\sigma}}\left\langle\cos \left(\Delta_{\mathbf{r}} \theta^{>}\right)\right\rangle_{>}\left[1-\cos \left(\Delta_{\mathbf{r}} \theta^{<}\right)\right]
$$

(from now on we omit the $r>a$ condition in the integral over $r$ ). On the other hand, $\left\langle\cos \left(\Delta_{\mathbf{r}} \theta^{>}\right)\right\rangle_{>}=e^{-\frac{1}{2}\left\langle(\theta(\mathbf{r})-\theta(0))^{2}\right\rangle_{>}}$ and

$$
\frac{1}{2}\left\langle(\theta(\mathbf{r})-\theta(0))^{2}\right\rangle_{>}=\int_{\Lambda>q>\Lambda e^{-d \ell}} \frac{d^{2} q}{(2 \pi)^{2}} \frac{1-\cos (\mathbf{q} \cdot \mathbf{r})}{J_{\ell} q^{2}}=\frac{d \ell}{2 \pi J_{\ell}}\left(1-\mathcal{J}_{0}(\Lambda r)\right)
$$

where $\mathcal{J}_{0}(x)$ is the zeroth-order Bessel function of the first kind. Then, introducing $\eta_{\mathrm{sr}}(J)=\frac{1}{2 \pi J_{\ell}}$, the exponent of the correlations at the cutoff scale $\ell$, we have:

$$
\begin{aligned}
\left\langle\cos \left(\Delta_{\mathrm{r}} \theta^{>}\right)\right\rangle_{>} & =e^{-\eta_{\mathrm{sr}}\left(J_{\ell}\right) d \ell\left(1-\mathcal{J}_{0}(\Lambda r)\right)} \\
& =1-\eta_{\mathrm{sr}}\left(J_{\ell}\right) d \ell+\eta_{\mathrm{sr}}\left(J_{\ell}\right) d \ell \mathcal{J}_{0}(\Lambda r)
\end{aligned}
$$

up to second order corrections. The first two terms provide an anomalous dimension of the coupling $g_{\ell+d \ell}=$ $g_{\ell} e^{-\eta_{\mathrm{sr}}\left(J_{\ell}\right) d \ell}$, as expected, while the last one modifies the power-law dependence on $r$ of the long-range term:

$$
\begin{aligned}
\left\langle S_{g}\right\rangle_{>} & =\frac{1}{2} \int d^{2} x\left\{\int \frac{d^{2} r}{r^{2+\sigma}} g e^{-\eta_{\mathrm{sr}}\left(J_{\ell}\right) d \ell}\left[1-\cos \left(\Delta_{\mathbf{r}} \theta^{<}\right)\right]\right. \\
& \left.+g \eta_{\mathrm{sr}}\left(J_{\ell}\right) d \ell \int \frac{d^{2} r}{r^{2+\sigma}} \mathcal{J}_{0}(\Lambda r)\left[1-\cos \left(\Delta_{\mathbf{r}} \theta^{<}\right)\right]\right\} .
\end{aligned}
$$

Let us now examine the last term of the above equation. This can be seen as an interaction term of the original $X Y$ form. Since $\mathcal{J}_{0}(x) \sim x^{-1 / 2} \cos (x-\pi / 4)$ for large $x$, the new coupling decays faster than the original and has an oscillating behavior, which provides a natural cutoff for $r \sim \Lambda^{-1}$. It is then reasonable to approximate this with a short-range coupling of the form $|\nabla \theta|^{2}$. The simplest way is to replace $1-\cos \left(\Delta_{r} \theta\right) \approx \frac{1}{2}\left(\mathbf{r} \cdot \nabla_{\mathbf{x}} \theta\right)^{2}$ and observe that

$$
\int \frac{d^{2} r}{r^{2+\sigma}} \mathcal{J}_{0}(\Lambda r)\left(\mathbf{r} \cdot \nabla_{\mathbf{x}} \theta^{<}\right)^{2}=\pi\left|\nabla_{\mathbf{x}} \theta^{<}\right|^{2} \int_{a}^{\Lambda^{-1}} d r r^{1-\sigma} \mathcal{J}_{0}(\Lambda r)
$$

For $\sigma>\frac{1}{2}$, we can neglect the cutoff and, with the substitution $r=a u$, we can express the correction in the action as

$$
\frac{c_{\sigma}}{2}\left(g_{\ell} a^{2-\sigma}\right) \eta_{\mathrm{sr}}\left(J_{\ell}\right) d \ell \int d^{2} x\left|\nabla_{\mathbf{x}} \theta^{<}\right|^{2}
$$

where $c_{\sigma}=\frac{\pi}{2} \int_{1}^{\infty} d u u^{1-\sigma} \mathcal{J}_{0}(2 \pi u)>0$. The integral is actually ill-defined for $\sigma<\frac{1}{2}$ signaling that our approximation breaks down. Let us notice however that the precise value of the coefficient is not important for our analysis. Moreover, it should be noticed that this entire procedure is only reliable for $\sigma>7 / 4$, where part of the BKT fixed points line remains stable and furnishes a viable expansion point, see the discussion in the main text. Up to the first order in $g$, then the integration of the fast modes gives the corrections:

$$
\begin{aligned}
& d g=-\eta_{\mathrm{sr}}\left(J_{\ell}\right) g_{\ell} d \ell \\
& d J=c_{\sigma} \eta_{\mathrm{sr}}\left(J_{\ell}\right)\left(g_{\ell} a^{2-\sigma}\right) d \ell .
\end{aligned}
$$


In order to obtain a theory with the same cutoff scale, we have to do the replacement $\mathbf{x} \rightarrow \mathbf{x} e^{-d \ell}$ in the action. This modifies the couplings $g, J$ by their own bare length dimension, i.e. $2-\sigma$ and 0 respectively:

$$
\begin{aligned}
& d g=\left(2-\sigma-\eta_{\mathrm{sr}}\left(J_{\ell}\right)\right) g_{\ell} d \ell \\
& d J=c_{\sigma} \eta_{\mathrm{sr}}\left(J_{\ell}\right)\left(g_{\ell} a_{0}^{2-\sigma}\right) d \ell .
\end{aligned}
$$

In turn, one finally obtains the RG equations:

$$
\begin{aligned}
& \frac{d g}{d \ell}=\left(2-\sigma-\eta_{\mathrm{sr}}\left(J_{\ell}\right)\right) g_{\ell} \\
& \frac{d J}{d \ell}=c_{\sigma} \eta_{\mathrm{sr}}\left(J_{\ell}\right) g_{\ell},
\end{aligned}
$$

i.e. Eqs. (7) of the main text (we absorbed the constant ultraviolet cutoff $a^{2-\sigma}$ in the definition of $c_{\sigma}$ ).

\section{Magnetization in the low-temperature phase}

We will now derive the scaling behavior (10) given in the main text for the magnetization near $T_{c}$, for $T \rightarrow T_{c}^{-}$. We start from the Gaussian theory, Eq. (9) of the main text, describing the low temperature phase of the theory in the infrared (IR). Being the theory Gaussian, it is $m=\langle\cos \theta(\mathbf{x})\rangle=e^{-\frac{1}{2}\left\langle\theta^{2}(\mathbf{x})\right\rangle}$. Being

$$
\left\langle\theta^{2}(\mathbf{x})\right\rangle=\int_{q<2 \pi / a} \frac{d^{2} q}{\left(2 \pi^{2}\right)} \frac{1}{\bar{g} q^{\sigma}} \sim \frac{1}{\bar{g} a^{\sigma-2}} .
$$

we find

$$
m=e^{-A / \bar{g}}
$$

where $A$ is a non-universal constant. Now, from the flow equations 229, we find:

$$
g_{\ell}=g e^{(2-\sigma) \ell} e^{-\int \eta_{\mathrm{sr}}\left(J_{\ell}\right) d \ell},
$$

which is reliable as long as $g_{\ell}$ is small. Let us consider a trajectory which runs very close to the separatrix which, according to Eq. (8) of the main text, is described by the trajectory $g=\frac{\pi(2-\sigma)}{c_{\sigma}}\left[\left(J-J_{\sigma}\right)^{2}+k\right]$ with $k \rightarrow 0^{+}$. Let us consider a point in the flow $\ell^{*}$ such that $g\left(\ell^{*}\right)$ is small and $J\left(\ell^{*}\right)>J_{\sigma}$. Then:

$$
\int_{0}^{\ell^{*}} \eta_{\mathrm{sr}}\left(J_{\ell}\right) d \ell=\int_{J_{0}}^{\ell^{*}} \eta_{\mathrm{sr}}(J) \frac{d J}{\dot{J}}=c_{\sigma}^{-1} \int_{J_{0}}^{\ell^{*}} \frac{d J}{g(J)}=\pi(2-\sigma) \int_{J_{0}}^{J\left(\ell^{*}\right)} \frac{d J}{\left(J-J_{\sigma}\right)^{2}+k}
$$

By changing the value of the temperature, we have that $J_{0}$ crosses the separatrix $\left(k \rightarrow 0^{+}\right)$for some $J_{c}<J_{\sigma}$ that corresponds to the critical temperature $T_{c}$, and consequently $k \sim T_{c}-T$. Since in this case the integration interval on $J$ contains the second order singularity $J_{\sigma}$, we have that the integral diverges as $k^{-1 / 2}$ as $k \rightarrow 0^{+}$. Then we have

$$
g_{\ell^{*}} \sim e^{-B\left(T-T_{c}\right)^{-1 / 2}}
$$

where $B$ is a non universal constant. Since, as $k \rightarrow 0^{+}$, the trajectories corresponding to different values of $k$ run close in the parameter space, for large $g$ as well, we do not expect this scaling to be modified in the non-perturbative region. Finally, exploiting Eq. (31), one has the scaling:

$$
\ln m \sim-A e^{B\left(T-T_{c}\right)^{-1 / 2}}
$$

\section{Irrelevance of topological excitations in the low-temperature phase}

We start from the quadratic action of Eq. (9) of the main text, which describes the low temperature phase, we express it in terms of the Fourier transform of $\mathbf{v}(\mathbf{x})=\nabla \theta$

$$
S_{g}=\bar{g} \int \frac{d^{2} q}{(2 \pi)^{2}} q^{\sigma}|\theta(\mathbf{q})|^{2}=\bar{g} \int \frac{d^{2} q}{(2 \pi)^{2}} q^{\sigma-2}|\mathbf{v}(\mathbf{q})|^{2}
$$

We notice that circling around a topologically non-trivial region we have

$$
\oint \nabla \theta \cdot d \mathbf{r}=\oint \mathbf{v} \cdot d \mathbf{r}=2 \pi m_{e n c}
$$


were $m_{\text {enc }}$ is the sum of all the topological charges $m_{i}$ enclosed in the integration contour. This can be rephrased by saying that $\nabla \times \mathbf{v}(\mathbf{x})=2 \pi n(\mathbf{x})$, where $n(\mathbf{x})=\sum_{j} m_{j} \delta\left(x-x_{j}\right)$ is the vortex-density and $x_{j}$ correspond to the positions of the vortices. This can be further simplified if we introduce the dual $\mathbf{u}(\mathbf{x})$ of $\mathbf{v}(\mathbf{x})$, defined as $u_{j}=\epsilon_{j k} v_{k}$ where $\epsilon_{j k}$ is the fully antisymmetric tensor of rank 2 . We then find the condition

$$
\nabla \cdot \mathbf{u}(\mathbf{x})=2 \pi n(\mathbf{x})
$$

In turn, this can be solved in the Fourier space:

$$
\mathbf{u}(\mathbf{q})=\frac{2 \pi \mathbf{q}}{q^{2}} n(\mathbf{q})+\mathbf{u}_{\perp}(\mathbf{q})
$$

where $\mathbf{u}_{\perp}(\mathbf{q})$ is a generic function such that $\mathbf{q} \cdot \mathbf{u}_{\perp}(\mathbf{q})=0$ and which represent the topologically-trivial component of the field $\theta$. Now, since

$$
|\mathbf{v}(\mathbf{q})|^{2}=|\mathbf{u}(\mathbf{q})|^{2}=\frac{(2 \pi)^{2}}{q^{2}}|n(\mathbf{q})|^{2}+\left|\mathbf{u}_{\perp}(\mathbf{q})\right|^{2}
$$

we have that the action $S_{g}$ splits into the sum on the non-topological and topological part, the latter being:

$$
S_{\mathrm{top}}=\bar{g} \int d^{2} q q^{\sigma-4}|n(\mathbf{q})|^{2}
$$

Coming back to the real space we have:

$$
S_{\mathrm{top}}=\bar{g} \sum_{i j} m_{i} m_{j} G\left(\mathbf{r}_{i}-\mathbf{r}_{j}\right)
$$

with $G(\mathbf{x})=\int d^{2} q q^{\sigma-4} e^{i \mathbf{q} \cdot \mathbf{x}} \sim L^{2-\sigma}-x^{2-\sigma}, L$ being the system size. The first term in $G$ gives raise to a term proportional to $L^{2-\sigma} \sum_{i, j} m_{i} m_{j}=L^{2-\sigma}\left(\sum_{i} m_{i}\right)^{2}$ which, in the thermodynamic limit, ensures the neutrality of the gas of charges. We find then

$$
S_{\text {top }} \sim-\bar{g} \sum_{i j} m_{i} m_{j}\left|\mathbf{x}_{i}-\mathbf{x}_{j}\right|^{2-\sigma}
$$

As expected, this interaction is more binding than the logarithmic one for the short-range case. A simple entropyenergy argument shows that the charges will never unbound at any temperature: indeed the energetic cost of creating two far apart vortices grows like $\bar{g} L^{2-\sigma}$ while the entropy as $\ln L$ so that the free energy

$$
F \sim \ln L-\bar{g} T L^{2-\sigma}
$$

is always dominated by the interaction term for large enough $L$.

\footnotetext{
* ggiachet@sissa.it
}

[1] J. Zinn-Justin, 4th ed. (Oxford University Press, Oxford, 2002).

[2] J. M. Kosterlitz, Rev. Mod. Phys. 89, 040501 (2017).

[3] H. Kunz and C. E. Pfister, Communications in Mathematical Physics 46, 245 (1976).

[4] J. Sak, Phys. Rev. B 8, 281 (1973).

[5] E. Luijten and H. W. J. Blöte, Phys. Rev. Lett. 89, 1 (2002).

[6] T. Blanchard, M. Picco, and M. A. Rajabpour, EPL (Europhysics Letters) 101, 56003 (2013), arXiv:1211.6758 [condmat.stat-mech]

[7] P. Grassberger, Journal of Statistical Physics 153, 289 (2013), arXiv:1305.5940 [cond-mat.stat-mech].

[8] M. C. Angelini, G. Parisi, and F. Ricci-Tersenghi, Phys. Rev. E 89, 062120 (2014)

[9] T. Horita, H. Suwa, and S. Todo, Phys. Rev. E 95, 012143 (2017).

[10] E. Brezin, G. Parisi, and F. Ricci-Tersenghi, Journal of Statistical Physics 157, 855 (2014), arXiv:1407.3358 [cond-mat.statmech],

[11] N. Defenu, A. Trombettoni, and A. Codello, Phys. Rev. E 92, 052113 (2015).

[12] N. Defenu, A. Trombettoni, and S. Ruffo, Phys. Rev. B 94, 224411 (2016).

[13] C. Behan, L. Rastelli, S. Rychkov, and B. Zan, Phys. Rev. Lett. 118, 241601 (2017).

[14] E. Luijten, Ph.D. thesis, Technische Universiteit Delft (1997).

[15] N. Defenu, A. Codello, S. Ruffo, and A. Trombettoni, Journal of Physics A Mathematical General 53, 143001 (2020)

[16] A. Dutta and J. K. Bhattacharjee, Phys. Rev. B 64, 184106 (2001).

[17] N. Defenu, A. Trombettoni, and S. Ruffo, Phys. Rev. B 96, 104432 (2017).

[18] S. T. Bramwell and P. C. W. Holdsworth, Phys. Rev. B 49, 8811 (1994) 
[19] R. Savit, Rev. Mod. Phys. 52, 453 (1980)

[20] P. Minnhagen, Rev. Mod. Phys. 59, 1001 (1987)

[21] Z. Gulácsi and M. Gulácsi, Adv. Phys. 47, 1 (1998).

[22] D. J. Amit, Y. Y. Goldschmidt, and S. Grinstein, J. Phys. A: Math. Gen. 13, 585 (1980)

[23] D. C. Mattis, Physics Letters A 104, 357 (1984).

[24] A. Filinov, N. V. Prokof'ev, and M. Bonitz, Phys. Rev. Lett. 105, 070401 (2010)

[25] J. Villain, J. Phys. France 36, 581 (1975)

[26] J. V. José, L. P. Kadanoff, S. Kirkpatrick, and D. R. Nelson, Phys. Rev. B 16, 1217 (1977).

[27] J. N. V. N. Popov, 1st ed., Mathematical Physics and Applied Mathematics (Kluwer, 1983).

[28] V. L. Berezinskii, Sov. Phys. JETP 34, 610 (1972).

[29] J. M. Kosterlitz and D. J. Thouless, Journal of Physics C: Solid State Physics 6, 1181 (1973).

[30] J. M. Kosterlitz, J. Phys. C 7, 1046 (1974).

[31] J. V. José, ed., (World Scientific, Singapore, 2013).

[32] D. J. Bishop and J. D. Reppy, Phys. Rev. Lett. 40, 1727 (1978)

[33] J. Corson, R. Mallozzi, J. Orenstein, J. N. Eckstein, and I. Bozovic, Nature (London) 398, 221 (1999)

[34] I. Hetel, T. R. Lemberger, and M. Randeira, Nat. Phys. 3, 700 (2007)

[35] L. S. Bilbro, R. V. Aguilar, G. Logvenov, O. Pelleg, I. Božovič, and N. P. Armitage, Nat. Phys. 7, 298 (2011)

[36] J. Yong, M. J. Hinton, A. McCray, M. Randeria, M. Naamneh, A. Kanigel, and T. R. Lemberger, Phys. Rev. B 85, 180507 $(2012)$

[37] P. G. Baity, X. Shi, Z. Shi, L. Benfatto, and D. Popović, Phys. Rev. B 93, 024519 (2016)

[38] W. H. Nitsche, N. Y. Kim, G. Roumpos, C. Schneider, M. Kamp, S. Höfling, A. Forchel, and Y. Yamamoto, Phys. Rev. B 90, $205430(2014)$.

[39] Z. Hadzibabic, P. Krüger, M. Cheneau, B. Battelier, and J. Dalibard, Nature (London) 441, 1118 (2006)

[40] P. A. Murthy, I. Boettcher, L. Bayha, M. Holzmann, D. Kedar, M. Neidig, M. G. Ries, A. N. Wenz, G. Zürn, and S. Jochim, Phys. Rev. Lett. 115, 010401 (2015).

[41] N. Reyren, S. Thiel, A. D. Caviglia, L. F. Kourkoutis, G. Hammerl, C. Richter, C. W. Schneider, T. Kopp, A.-S. Rüetschi, D. Jaccard, M. Gabay, D. A. Muller, J.-M. Triscone, and J. Mannhart, Science 317, 1196 (2007).

[42] G. N. Daptary, S. Kumar, P. Kumar, A. Dogra, N. Mohanta, A. Taraphder, and A. Bid, Phys. Rev. B 94, 085104 (2016).

[43] A. M. R. V. L. Monteiro, D. J. Groenendijk, I. Groen, J. de Bruijckere, R. Gaudenzi, H. S. J. van der Zant, and A. D. Caviglia, Phys. Rev. B 96, 020504(R) (2017).

[44] C. Nisoli and A. R. Bishop, Phys. Rev. Lett. 112, 070401 (2014)

[45] M. Seul, L. R. Monar, L. O'Gorman, and R. Wolfe, Science 254, 1616 (1991)

[46] A. Mendoza-Coto and D. A. Stariolo, Phys. Rev. E 86, 051130 (2012).

[47] C. Monroe, W. C. Campbell, L. M. Duan, Z. X. Gong, A. V. Gorshkov, P. Hess, R. Islam, K. Kim, G. Pagano, P. Richerme, C. Senko, and N. Y. Yao, arXiv e-prints, arXiv:1912.07845 (2019), arXiv:1912.07845 [quant-ph]

[48] J. W. Britton, B. C. Sawyer, A. C. Keith, C. C. J. Wang, J. K. Freericks, H. Uys, M. J. Biercuk, and J. J. Bollinger, Nature 484, $489(2012)$

[49] M. A. Baranov, M. Dalmonte, G. Pupillo, and P. Zoller, arXiv e-prints , arXiv:1207.1914 (2012), arXiv:1207.1914 [condmat.quant-gas]

[50] R. Landig, L. Hruby, N. Dogra, M. Land ini, R. Mottl, T. Donner, and T. Esslinger, Nature 532, 476 (2016).

[51] T. Botzung, D. Hagenmüller, G. Masella, J. Dubail, N. Defenu, A. Trombettoni, and G. Pupillo, Phys. Rev. B 103, 155139 $(2021)$.

[52] P. Jurcevic, B. P. Lanyon, P. Hauke, C. Hempel, P. Zoller, R. Blatt, and C. F. Roos, Nature 511, 202 (2014).

[53] P. Richerme, Z.-X. Gong, A. Lee, C. Senko, J. Smith, M. Foss-Feig, S. Michalakis, A. V. Gorshkov, and C. Monroe, Nature 511, $198(2014)$

[54] J. Zhang, P. W. Hess, A. Kyprianidis, P. Becker, A. Lee, J. Smith, G. Pagano, I. D. Potirniche, A. C. Potter, A. Vishwanath, N. Y. Yao, and C. Monroe, Nature 543, 217 (2017)

[55] K. Baumann, C. Guerlin, F. Brennecke, and T. Esslinger, Nature 464, 1301 (2010).

[56] J. Rovny, R. L. Blum, and S. E. Barrett, Phys. Rev. Lett. 120, 180603 (2018).

[57] S. Choi, J. Choi, R. Landig, G. Kucsko, H. Zhou, J. Isoya, F. Jelezko, S. Onoda, H. Sumiya, V. Khemani, C. von Keyserlingk, N. Y. Yao, E. Demler, and M. D. Lukin, Nature 543, 221 (2017).

[58] A. Safavi-Naini, R. J. Lewis-Swan, J. G. Bohnet, M. Gärttner, K. A. Gilmore, J. E. Jordan, J. Cohn, J. K. Freericks, A. M. Rey, and J. J. Bollinger, Phys. Rev. Lett. 121, 040503 (2018)

[59] A. Keesling, A. Omran, H. Levine, H. Bernien, H. Pichler, S. Choi, R. Samajdar, S. Schwartz, P. Silvi, S. Sachdev, P. Zoller, M. Endres, M. Greiner, Vuletić, V. , and M. D. Lukin, Nature 568, 207 (2019)

[60] P. Hauke and L. Tagliacozzo, Phys. Rev. Lett. 111, 207202 (2013), arXiv:1304.7725 [quant-ph].

[61] D. Vodola, L. Lepori, E. Ercolessi, A. V. Gorshkov, and G. Pupillo, Phys. Rev. Lett. 113, 156402 (2014).

[62] M. F. Maghrebi, Z.-X. Gong, M. Foss-Feig, and A. V. Gorshkov, Phys. Rev. B 93, 125128 (2016)

[63] Z.-X. Gong, M. F. Maghrebi, A. Hu, M. Foss-Feig, P. Richerme, C. Monroe, and A. V. Gorshkov, Phys. Rev. B 93, 205115 $(2016)$.

[64] N. Defenu, T. Enss, and J. C. Halimeh, Phys. Rev. B 100, 014434 (2019).

[65] P. Uhrich, N. Defenu, R. Jafari, and J. C. Halimeh, Phys. Rev. B 101, 245148 (2020)

[66] A. Campa, T. Dauxois, D. Fanelli, and S. Ruffo, Physics of Long-Range Interacting Systems (Oxford Univ. Press, 2014).

[67] S. Romano, Nuovo Cimento B Serie 100, 447 (1987).

[68] Giachetti, Guido, Defenu, Nicolò, Ruffo, Stefano, and Trombettoni, Andrea, EPL 133, 57004 (2021)

[69] M. F. Maghrebi, Z.-X. Gong, and A. V. Gorshkov, Phys. Rev. Lett. 119, 023001 (2017)

[70] L. Benfatto, C. Castellani, and T. Giamarchi, in 40 Years of Berezinskii-Kosterliz-Thouless Theory, edited by J. V. José (World Scientific, Singapore, 2013). 
[71] C. Itzykson and J.-M. Drouffe, Statistical field theory. Volume 1, From Brownian motion to renormalization and lattice gauge theory (Cambridge, Cambridge University Press, 1989).

[72] M. Le Bellac, Quantum and statistical field theory (Oxford, Clarendon, 1991).

[73] J. V. José, L. P. Kadanoff, S. Kirkpatrick, and D. R. Nelson, Phys. Rev. B 16, 1217 (1977).

[74] H. Spohn and W. Zwerger, Journal of Statistical Physics 94, 1037 (1999), arXiv:cond-mat/9811219 [cond-mat.stat-mech].

[75] A. Kargol, Reports on Mathematical Physics 56, 379 (2005).

[76] A. Kargol, Journal of Statistical Mechanics: Theory and Experiment 2014, 10006 (2014)

[77] S. R. Shenoy and B. Chattopadhyay, Phys. Rev. B 51, 9129 (1995)

[78] G. Bighin, N. Defenu, I. Nándori, L. Salasnich, and A. Trombettoni, Phys. Rev. Lett. 123, 100601 (2019)

[79] P. G. Maier and F. Schwabl, Phys. Rev. B 70, 134430 (2004)

[80] A. Vasilyev, A. Tarkhov, L. Men'shikov, P. Fedichev, and U. Fischer, New Journal of Physics 16, 053011 (2014)

[81] F. Antenucci, M. Ibáñez Berganza, and L. Leuzzi, Phys. Rev. B 92, 014204 (2015)

[82] L. Jacobs and R. Savit, Annals of the New York Academy of Sciences 410, 281 (1983).

[83] N. Defenu, A. Trombettoni, and D. Zappalà, Nuclear Physics B 964, 115295 (2021)

[84] A. Raju, C. B. Clement, L. X. Hayden, J. P. Kent-Dobias, D. B. Liarte, D. Z. Rocklin, and J. P. Sethna, Phys. Rev. X 9, $021014(2019)$

[85] I. Maccari, N. Defenu, L. Benfatto, C. Castellani, and T. Enss, Phys. Rev. B 102, 104505 (2020)

[86] M. Ibáñez-Berganza and L. Leuzzi, Phys. Rev. B 88, 144104 (2013).

[87] F. Cescatti, M. Ibáñez-Berganza, A. Vezzani, and R. Burioni, Phys. Rev. B 100, 054203 (2019), arXiv:1905.06688 [condmat.stat-mech]

[88] A. Bermudez, L. Tagliacozzo, G. Sierra, and P. Richerme, Phys. Rev. B 95, 024431 (2017)

[89] T. Botzung, D. Hagenmüller, G. Masella, J. Dubail, N. Defenu, A. Trombettoni, and G. Pupillo, Phys. Rev. B 103, 155139 $(2021)$. 